PSFC/JA-06-10

\title{
Measured dependence of nuclear burn region size on implosion parameters in inertial confinement fusion experiments
}

\author{
F. H. Séguin, ${ }^{1}$ J. L. DeCiantis, ${ }^{1}$ J. A. Frenje, ${ }^{1}$ C. K. Li, ${ }^{1}$ J. R. \\ Rygg, ${ }^{1}$ C. D. Chen, ${ }^{1}$ R. D. Petrasso ${ }^{1}$, J. A. Delettrez, ${ }^{2}$ S. P. \\ Regan, ${ }^{2}$ V. A. Smalyuk, ${ }^{2}$ V. Yu. Glebov, ${ }^{2}$ J. P. Knauer, ${ }^{2}$ \\ F. J. Marshall, ${ }^{2}$ D. D. Meyerhofer, ${ }^{2}$ S. Roberts, ${ }^{2}$ \\ T. C. Sangster, ${ }^{2}$ C. Stoeckl, ${ }^{2}$ K. Mikaelian, ${ }^{3}$ H. S. Park, ${ }^{3}$ \\ H. F. Robey, ${ }^{3}$ and R. E. Tipton ${ }^{3}$ \\ ${ }^{1}$ Plasma Science and Fusion Center, Massachusetts Institute of \\ Technology, Cambridge, Massachusetts, 02139 \\ ${ }^{2}$ Laboratory for Laser Energetics, University of Rochester, \\ Rochester, New York, 14623 \\ ${ }^{3}$ Lawrence Livermore National Laboratory, Livermore, California \\ 94550
}

1 July 2006

This work was supported in part by U.S. Department of Energy Contract \#DEFG03-99SF21782, LLE subcontract \#PO410025G, and LLNL subcontract \#B313975.

Accepted for publication in Physics of Plasmas 


\title{
Measured dependence of nuclear burn region size on implosion parameters in inertial confinement fusion experiments
}

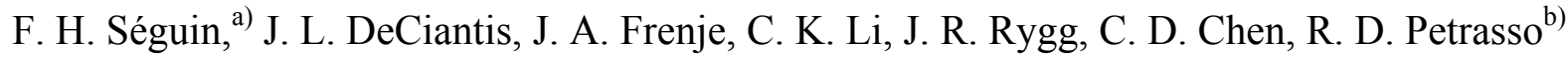 \\ Plasma Science and Fusion Center, Massachusetts Institute of Technology, \\ Cambridge, Massachusetts, 02139
}

J. A. Delettrez, S. P. Regan, V. A. Smalyuk, V. Yu. Glebov, J. P. Knauer, F. J. Marshall, D.

D. Meyerhofer ${ }^{\text {c) }}$, S. Roberts, T. C. Sangster, C. Stoeckl

Laboratory for Laser Energetics, University of Rochester, Rochester, New York, 14623

K. Mikaelian, H. S. Park, H. F. Robey, R. E. Tipton

Lawrence Livermore National Laboratory, Livermore, California 94550

\begin{abstract}
Radial profiles of nuclear burn in directly-driven, inertial-confinement-fusion implosions have been systematically studied for the first time using a proton emission imaging system sensitive to energetic $14.7-\mathrm{MeV}$ protons from the fusion of deuterium (D) and 3-helium $\left({ }^{3} \mathrm{He}\right)$ at the OMEGA laser facility [T. R. Boehly et al., Opt. Commun. 133, 495 (1997)]. Experimental parameters that were varied include capsule size, shell composition and thickness, gas fill pressure, and laser energy. Clear relationships have been identified between changes in a number of these parameters and changes in the size of the burn region, which we characterize here by the median "burn radius" $R_{\text {burn }}$ containing half of the total $\mathrm{D}^{3} \mathrm{He}$ reactions. Different laser and capsule parameters resulted in burn radii varying from 20 to 80 $\mu \mathrm{m}$. For example, reducing the $\mathrm{D}^{3} \mathrm{He}$ fill pressure from 18 to $3.6 \mathrm{~atm}$ in capsules with $20-\mu \mathrm{m}$ thick $\mathrm{CH}$ shells resulted in the mean burn radius changing from $31 \mu \mathrm{m}$ to $25 \mu \mathrm{m}$; this reduction is attributed to increased fuel-shell mix for the more unstable 3.6-atm implosions rather than to increased convergence, because total areal density didn't change very much. Fuel-shell-interface radii estimated from hard (4-5 keV) x-ray images of some of the same implosions were observed to closely track the burn radii. Simulated burn radii produced with 1-D codes agree fairly well with measurements for glass-shell capsules, but are systematically smaller than measurements for $\mathrm{CH}$-shell capsules. A search for possible sources of systematic measurement error that could account for this discrepancy has been unsuccessful. Possible physical sources of discrepancies are mix, hydrodynamic instabilities and/or preheat not included in the 1-D code. Since measured burn-region sizes indicate where fusion actually occurs as a consequence of all the complicated processes that affect capsule implosion dynamics, they provide exacting tests of simulations.
\end{abstract}

a)seguin@mit.edu

b) Also Visiting Senior Scientist, Laboratory for Laser Energetics, University of Rochester.

c) Also Departments of Mechanical Engineering, Physics and Astronomy, University of Rochester.

Byline: ICF burn region measurements 


\section{INTRODUCTION}

Images of the nuclear burn regions in inertial confinement fusion (ICF) capsules are important for fully assessing the combined results of all the complicated processes that affect capsule implosion dynamics; these processes, including drive, preheat, instabilities and mix, must be understood and controlled in order to achieve ignition and energy generation. ${ }^{1-3}$ In the direct-drive approach to ICF, a spherical capsule containing fuel is compressed and heated by direct illumination of laser beams focused on the capsule surface in a nominally uniform fashion. ${ }^{2}$ Hydrodynamic instabilities, drive characteristics, and capsule structure affect the performance of these implosions, ${ }^{2-10}$ ultimately determining the size, symmetry, and yield of the nuclear burn region. This article, the third in a series about proton emission imaging, ${ }^{11-12}$ presents the first comprehensive studies of $\mathrm{D}^{3} \mathrm{He}$ burn region sizes in nominally symmetric direct-drive implosions with diverse capsule and drive conditions. Radial burn profiles and total yields are obtained from measurements of energetic 14.7-MeV protons from the fusion of deuterium (D) and 3-helium $\left({ }^{3} \mathrm{He}\right)$ using methods described in Refs. 11 and 12 and in the Appendix of this article. Complementary data from $\mathrm{x}$-ray images, ${ }^{14-17}$ proton spectrometers, ${ }^{18-23}$ and clean 1-D simulations ${ }^{24,25}$ are used in interpreting the burn-region sizes and their implications. Asymmetric burn distributions and their relationships to implosion conditions are described in Refs. 11 and 12, and tests of the fidelity of the reconstructed images are discussed in Refs. 11 and 12. Burn images have previously been made of deuterium-tritium-filled capsules using $14.1-\mathrm{MeV}$ neutrons; ${ }^{8,26-29} 3-\mathrm{MeV}$ protons $^{30-32}$ or $3.5-$ $\mathrm{MeV}$ alpha particles, ${ }^{32}$ but for a more limited range of implosion types (see also other papers cited in Ref. 11).

Section II describes the drive and capsule parameters studied, along with general information about the proton emission imaging cameras and the other diagnostics used. Section III provides an overview of the analysis of proton imaging data, together with examples from two implosions that have dramatically different burn regions. Similarities and differences between nuclear burn images and x-ray images are discussed; and important connections between burn profiles, areal density $(\rho R)$, and clean 1-D simulations are made. Section IV summarizes the results obtained when capsule and drive conditions were systematically altered to modify the burn region. The dependence of the burn radius on shell thickness, gas pressure, laser energy, and shell type were investigated for a large set of implosions, and evidence of the presence of mix is discussed. Section V summarizes the results and future work, and an Appendix provides detailed information about methods of calculating radial burn profiles from penumbral images (including neutron images).

\section{EXPERIMENTAL CONDITIONS}

To explore the range of burn regions associated with different kinds of symmetrically driven implosions, and to reveal the complicated physics, such as preheat, mix, drive efficiency, and core distortions, a wide variety of implosions was examined on the OMEGA laser system. $^{33}$ OMEGA is a 60 -beam, frequency-tripled UV $(0.35 \mu \mathrm{m})$ laser capable of delivering up to $30 \mathrm{~kJ}$ of laser energy in a variety of pulse shapes. The individual laser beams were smoothed with distributed phase plates (DPPs), ${ }^{34}$ two-dimensional smoothing by spectral dispersion with a bandwidth of $1.0 \mathrm{THz},{ }^{35,36}$ and polarization smoothing using birefringent wedges. $^{37}$ Two types of DPPs (SG3 and SG4) were used in the experiments described here, producing different beam intensity profiles. ${ }^{38}$ Only 1-ns square laser pulses were used to directly illuminate the capsule. The beam-to-beam energy imbalance was typically less than $4 \% \mathrm{rms}$. 
The capsules used had either glass $\left(\mathrm{SiO}_{2}\right)$ shells 1.8 to $2.3 \mu \mathrm{m}$ thick, filled with 18 -atm $\mathrm{D}^{3} \mathrm{He}$ gas, or plastic $(\mathrm{CH})$ shells 17 to $24 \mu \mathrm{m}$ thick, filled with 3.6 or 18 atm $\mathrm{D}^{3} \mathrm{He}$ gas. The glass-shell implosions used SG3 DPPs, while plastic shell implosions used SG4 DPPs except where noted. Initial capsule radii were nominally $470 \mu \mathrm{m}$ for the SG3 DPPs and $430 \mu \mathrm{m}$ for SG4 DPPs.

Proton core imaging system (PCIS) cameras ${ }^{11,12,12}$ imaged the time-integrated $\mathrm{D}^{3} \mathrm{He}$ proton emission distribution from up to three nearly orthogonal directions simultaneously. These are penumbral imaging cameras, each consisting of a round imaging aperture that is significantly larger than the size of the $\mathrm{D}^{3} \mathrm{He}$ burn region and a detector pack comprised of several ranging filters and CR-39 charged-particle detectors. ${ }^{12,19}$ The distances from the implosion to the imaging aperture and from the imaging aperture to the detector pack $\left(L_{1}\right.$ and $L_{2}$, respectively) determine the geometric magnification $M \equiv L_{2} / L_{1}$. Aperture diameters of $600 \mu \mathrm{m}$ and $2000 \mu \mathrm{m}$ were used. $L_{1}$ was typically $3 \mathrm{~cm}$ and $M$ varied from 8 to 20 . The energetic protons that pass through the aperture are detected with $100 \%$ efficiency in the CR39 , as long as the detector has filtering that slows incoming protons down to the CR-39 sensitivity range of about 0.5 to $8 \mathrm{MeV}$.

X-ray framing cameras were used to obtain $4-5 \mathrm{keV}$ x-ray emission images ${ }^{14-16}$ at 58 ps time intervals using $12 \mathrm{x}$ magnification and 40-ps integration times. At the time of peak proton production, the x-ray images represent primarily continuum emission from the heated inner portion of the shell material and can be used to estimate the radius of the fuel-shell interface as described in ref. 14.

Up to five proton spectrometers ${ }^{19}$ were used simultaneously to obtain time-integrated measurements of the $\mathrm{D}^{3} \mathrm{He}$ proton spectrum. These spectra are used to determine the total areal density $<\rho \mathrm{R}>>^{19,23,39}$ using the downshift from the $14.7-\mathrm{MeV}$ birth energy. ${ }^{18-22,39}$ In these experiments the measured total $\rho R$ comprises both the shell $\rho R$ and the fuel $\rho R$, but is usually dominated by the shell. ${ }^{19,23,39}$ For given capsule shell and laser conditions, $\rho \mathrm{R}$ provides a measure of shell convergence since, all else being equal, $\rho R$ scales as the inverse of the square of the shell radius at the time of burn. Important to this analysis, PCIS and spectrometers typically give the same $\mathrm{D}^{3} \mathrm{He}$ yield to within the observed proton-yield asymmetry of $15-20 \% \mathrm{rms}^{19}$

\section{DATA ANALYSIS}

\section{A. Finding the nuclear burn radius $\boldsymbol{R}_{\text {burn }}$}

The proton emission imaging cameras produce time-integrated penumbral images that are processed so as to produce either a 2-dimensional image of the burn-region surface brightness in $\mathrm{D}^{3} \mathrm{He}$ reactions per unit area, $B(r, \phi)$, or a 1-dimensional radial profile of the local number of $\mathrm{D}^{3} \mathrm{He}$ reactions per unit volume, $S(r)$, which corresponds to an average over angles. The 2$\mathrm{D}$ analysis technique is extremely useful for studying low-mode deviations from spherical symmetry, and simultaneous views of the burn region from three orthogonal directions can provide measurements of 3-dimensional structure; this approach has been used to study the important effects of asymmetric laser drive and asymmetric shell structure on implosion symmetry. ${ }^{12}$ A disadvantage of 2-D image reconstruction is that it always requires significant smoothing for control of statistical noise, as described in detail in ref. 11; for the types of implosions studied at OMEGA, $\mathrm{D}^{3} \mathrm{He}$ burn region sizes and yields typically require smoothing that limits spatial resolution to the range $15-30 \mu \mathrm{m}$. If it is desired to make an accurate measurement of characteristic burn region size for nearly-symmetric implosions, it is 
advantageous to use the 1-D approach, which typically results in statistical measurement uncertainties of a few $\mu \mathrm{m}$. Since the object of this paper is to study burn region size, we will use 1-D analysis here and discuss 2-D results elsewhere. ${ }^{12}$

The 1-D reconstruction approach utilizes the relationship between $S(r)$ and the radial derivative $\mathrm{d} N / \mathrm{d} R$ of the penumbral image values $N$ (proton tracks per unit area on the detector); $\mathrm{d} N / \mathrm{d} R$ is equivalent to a set of line integrals through the surface brightness of the $\mathrm{D}^{3} \mathrm{He}$ burn region as discussed in detail in ref. 11 and in the Appendix. Of two 1-D methods described in the Appendix, we will use in this paper the method of fitting $\mathrm{d} N / \mathrm{d} R$ with a family of functions that correspond either analytically or numerically to a family of local burn profile shapes. ${ }^{11}$ The radial profile $S(r)$ of the proton source in reactions per unit volume is represented by a member of the family of super Gaussians and sub Gaussians

$$
S(r)=S_{0} e^{-\left(r / r_{0}\right)^{2 / p}},
$$

where $p$ is a "peakedness" shape parameter and $r_{0}$ is a measure of burn radius; the median radius $R_{\text {burn }}$ containing half of the total local emission is actually used rather than $r_{0}$ to characterize the burn region size, because it can be determined much more accurately and is nearly independent of the emission profile shape (see the Appendix). The burn profile parameters and geometric parameters are then varied in order to generate the best fit between measured and predicted $\mathrm{d} N / \mathrm{d} R$.

Figure 1 shows sample data from two very different implosions involving capsules whose shells are 2- $\mu$ m-thick glass (Fig. 1a) and 20- $\mu$ m-thick plastic (Fig. 1e). The plotted data represent $\mathrm{d} N / \mathrm{d} R$ from the azimuthally-averaged penumbral images, with error bars representing statistical uncertainties. For each data set, a best fit (solid line) was found and used to determine the absolute radial profile of burn and its characteristic radius $R_{\text {burn }}$ (Figs. 1c and $1 \mathrm{~g}$ ) as well as the absolute radial profile of surface brightness $B(r)$ (Figs. $1 \mathrm{~d}$ and $1 \mathrm{~h}$ ). The values of $R_{\text {burn }}$ were $29 \pm 2.5 \mu \mathrm{m}$ for the plastic-shell implosion, and $54 \pm 2 \mu \mathrm{m}$ for the glassshell implosion (where the errors quoted here and throughout reflect statistical uncertainties). As discussed in the Appendix, the shapes of the radial profiles have their largest uncertainties at $r=0$, and the values of $R_{\text {burn }}$ are determined much more accurately than the shape parameter.

While multiple imaging cameras are generally used to study implosion asymmetry, a single camera provides enough information to calculate a 1-D emission profile of a nominally-symmetric capsule implosion. When data from more than one camera were available for an individual implosion studied in this paper, the images were analyzed separately and the values of $R_{\text {burn }}$ were averaged.

\section{B. Comparing nuclear burn data with $\mathrm{x}$-ray data}

Since x-ray imaging has been a standard diagnostic technique for decades, it is important to compare x-ray and fusion-burn profiles even though they have very different sensitivities to plasma processes and parameters. They provide valuable and complementary spatial information. The fusion burn profile $S(r)$ represents the time-integrated spatial distribution of the nuclear reaction rate

$$
R R=N_{D^{3} N^{3}}\langle\sigma v\rangle_{D^{3} \mathrm{He}},
$$

where $N_{D}$ and $N_{3 H e}$ are the $\mathrm{D}$ and ${ }^{3} \mathrm{He}$ ion number densities, and $\langle\sigma v\rangle$ is the reaction rate for the $\mathrm{D}-{ }^{3} \mathrm{He}$ reaction. In contrast, $\mathrm{x}$-ray images primarily record emissions from heated $\mathrm{CH}$ near the fuel-shell interface. ${ }^{14}$ In addition, the burn data are time integrated while the x-ray images are gated with a 40-ps window ${ }^{16}$ (and show slightly decreasing size during the $\sim 150$ ps burn interval). Figure 2 provides a comparison between burn data and an x-ray image taken approximately at peak burn time for shot 35176 (analyzed in Fig. 1. Although the 
image of x-ray surface brightness itself (Fig. 2b) isn't quite symmetric, its azimuthallyaveraged radial profile allows us to estimate that the fuel-shell interface was located approximately at the radius $R_{\text {xray }}=32 \pm 5 \mu \mathrm{m}$ where the brightness peaks before decreasing with increasing radius. ${ }^{14}$ The value of $R_{\text {burn }}$ was $29 \pm 2.5 \mu \mathrm{m}$; considering the different nature of the two kinds of data and the ambiguities as to exactly how they should be compared, the two measurements appear approximately consistent with each other. We will see in Sec. IV that the x-ray and nuclear burn profile data respond in a similar fashion to changes in the experimental conditions for a variety of implosions.

\section{Comparing nuclear burn data and $x$-ray data with 1-D simulations}

The burn data and x-ray data can also be compared to clean 1-D simulations, ${ }^{24,25}$ keeping in mind the facts that these simulations don't properly model such important physical processes as fuel-shell mix, preheat, or any type of implosion asymmetry, and that they nearly always overestimate the nuclear burn yield ${ }^{4}$. In Fig. 2 the measured local burn profile and the $\mathrm{x}$-ray profile for the plastic-shell implosion 35176 are compared to simulations. The predicted value of $R_{\text {xray }}$ is slightly smaller than the measured value (by about $15 \%$ ). The predicted profile of $\mathrm{D}^{3} \mathrm{He}$ burn is quite similar in shape to the measured profile in the core (although the measured shape uncertainty is large there, as shown in Fig. 1g), but the measured emission values are considerably higher than predicted at larger radii where they contribute heavily to the yield-weighted $R_{\text {burn }}$ : the predicted $R_{\text {burn }}$ is about $25 \%$ smaller than the measured value. On the other hand, there are other indications that the 1-D predictions aren't exactly right: the predicted yield was about $150 \%$ higher than the measured value, while the predicted shell areal density was $26 \%$ larger than the measured value (indicating that the shell did not converge radially as much as predicted). It will be seen in Sec. IV that measured values of $R_{\text {burn }}$ are uniformly larger than simulated values for the $\mathrm{CH}$-shell implosions studied here, but that measured and predicted values agree fairly well for a wide range of glass-shell implosions (which have different implosion dynamics ${ }^{40}$ ). Possible explanations of this measurement/simulation discrepancy in terms of either systematic measurement errors (which we have no evidence of ${ }^{41}$ ) or physics not included in 1-D simulations (mix, preheat, and hydrodynamic instabilities) are discussed in Sec. V.

\section{THE DEPENDENCE OF $\boldsymbol{R}_{\text {burn }}$ ON LASER AND CAPSULE PARAMETERS}

Correlations between $R_{\text {burn }}$ and the capsule and drive conditions allow an elucidation of some basic implosion dynamics. Systematic studies that examine the dependence of $R_{\text {burn }}$ on laser drive energy, capsule shell material and thicknesses, capsule fill pressure, and DPP type are presented here. These external parameters are often strongly correlated with one or more fundamental quantities or processes such as ion temperature, capsule convergence, fuel density, and fuel-shell mix. For that reason, attempts were made to change only one external parameter at a time in experiments whenever possible.

\section{A. Laser energy}

Figure 3a shows the effect of increasing laser energy, for 1-ns square pulses, when irradiating thin-glass-shell capsules (Fig. 3a, open diamonds). As the energy was increased from $6 \mathrm{~kJ}$ to $23 \mathrm{~kJ}, R_{\text {burn }}$ increased from about $35 \mu \mathrm{m}$ to $80 \mu \mathrm{m}$. For capsules with $19-20 \mu \mathrm{m}$ $\mathrm{CH}$ shells and 18 -atm $\mathrm{D}^{3} \mathrm{He}$ fill, $R_{\text {burn }}$ was about $30 \mu \mathrm{m}$; data are not yet available for illustrating variations with laser energy. The change in yield-weighted ion temperature 
$<T_{\text {ion }}>^{42}$ probably dominates the change in $R_{\text {burn }}$ for the glass-shell capsules, as illustrated in Fig. $3 \mathrm{~b}$ where the data of $3 \mathrm{a}$ are replotted as a function of $\left\langle T_{\text {ion }}>\right.$. Increases in $\left\langle T_{\text {ion }}>\right.$ should result in larger $R_{\text {burn }}$, since the $\mathrm{D}^{3} \mathrm{He}$ reactivity is extremely sensitive to the ion temperature. Figure $3 \mathrm{c}$ replots the data of $3 \mathrm{a}$ together with the corresponding $R_{\text {burn }}$ values from 1-D simulations (triangles). For the glass shells, 1-D simulations agree fairly well with $R_{\text {burn }}$ measurements and show the same variation with laser energy; for the plastic shells, the 1-D values are uniformly lower than the measured $R_{\text {burn }}$ values.

\section{B. Capsule fill pressure}

The effects of fill-pressure changes on $R_{\text {burn }}$ for $\mathrm{CH}$-shell implosions were studied systematically (Fig. 4). Figure 4 a shows $R_{\text {burn }}$ plotted as a function of measured $\langle\rho R>$ for fill pressures of $3.6 \mathrm{~atm}$ (open diamonds) and $18 \mathrm{~atm}$ (solid diamonds). $R_{\text {burn }}$ is consistently larger for the higher fill pressure. Figure $4 \mathrm{~b}$ shows the averages of the measured values ${ }^{43}$ for all implosions of each fill pressure in Fig. $4 \mathrm{a}$; the plotted values are

and

$$
<R_{\text {burn }}(18 \mathrm{~atm})>=30.6 \pm 0.3 \mu \mathrm{m}
$$

$$
<R_{\text {burn }}(3.6 \mathrm{~atm})>=24.8 \pm 0.8 \mu \mathrm{m},
$$

where the quoted errors reflect only the statistical uncertainties and do not include any other possible systematic errors. Figure $4 \mathrm{~b}$ also shows corresponding values of $R_{\text {xray }}$ for which we have data. The same trend is seen in both $R_{\text {burn }}$ and $R_{\text {xray. }}$. Figure $4 \mathrm{c}$ compares values of $R_{\text {burn }}$ and $\langle\rho R>$ from 1-D simulations (solid triangles for 18 atm and open triangles for $3.6 \mathrm{~atm}$ ) to the data. While the measured $R_{\text {burn }}$ values are all larger than predicted (as for all other plasticshell implosions studied here), there are other important differences between simulations and measurements. The 1-D simulations predict that a reduction in fill pressure from 18 atm to 3.6 atm should result in a large increase in $\rho R$ (by $\sim 100 \%$ ), due to increased radial convergence of the shell material, accompanied by a substantial decrease in $R_{\text {burn }}$ (by $\sim 50 \%$ ), largely due to a corresponding decrease in the core size. In contrast, the data indicate a much smaller increase in $\rho R$ (by $\sim 10 \%$ ), implying little change in radial convergence of the bulk of the shell material, and a moderate decrease in $R_{\text {burn }}$ (by $\sim 20 \%$ ).

These results agree quite well with data and interpretations published by C.K. Li et al. ${ }^{4}$ for implosions of plastic-shell capsules with DT fills of various pressures. For laser conditions and shell thicknesses similar to those examined here, it was concluded that the experimental radial convergence increased only slightly when the pressure was reduced from $\sim 18$ to $3 \mathrm{~atm}$, in distinct contrast to $1-\mathrm{D}$ calculations, exactly as seen here; it was suggested that the failure to achieve higher radial shell convergence with low fill pressure was probably due to fuel-shell mix, which converts some of the kinetic energy of radial shell movement to lateral motion. This is also consistent with the present data, which show a significant reduction in measured $R_{\text {burn }}$ when the pressure was decreased in spite of little change in the compressed shell radius; if the radius of most of the shell is held fixed, cooling and dilution of the outer fuel region due to the mixing in of a small amount of cooler shell material would reduce the number of $\mathrm{D}^{3} \mathrm{He}$ reactions there and reduce $R_{\text {burn }}$. The 1-D simulations predict a much larger decrease in $R_{\text {burn }}$ at lower fill pressure without invoking mix, but this is because they predict a much larger increase in radial convergence than is measured.

Finally, it seems plausible that the increased scatter of $R_{\text {burn }}$ at lower pressures may reflect decreased stability for those implosions. As shown in Fig. 4a, the standard deviation in the 3.6-atm data is larger than that for the 18 -atm data (by the ratio of $3.6 \mu \mathrm{m}$ to $1.4 \mu \mathrm{m}$ ). 


\section{Distributed Phase Plates}

An important goal of the OMEGA program is to improve the single and overlapping beam uniformity of the laser. As a step in that direction, the older SG3 DPPs were recently replaced with SG4 DPPs that result in a flatter on-target beam intensity $\left(\propto e^{-(r / 353 \mu m)^{4.1}}\right.$, where $r$ is radius from beam center, rather than $e^{-(r / 308 \mu m)^{2.2}}$ with SG3). ${ }^{38}$ To adjust for a reduction in the new spot size, the capsule radii were also reduced from $\sim 470$ to $430 \mu \mathrm{m}$. Figure 5 shows the effect of these changes upon $R_{\text {burn }}$ for several shots, plotted as a function of $\rho R$. $R_{\text {burn }}$ is larger for SG3 data than for SG4 data. The average measurement values ${ }^{43}$ of all implosions shown in Fig. 5a are

and

$$
<R_{\text {burn }}(\mathrm{SG} 3)>=37.1 \pm 0.8 \mu \mathrm{m}
$$

$$
<R_{\text {burn }}(\mathrm{SG} 4)>=30.5 \pm 0.3 \mu \mathrm{m}
$$

and are plotted in Fig. 5b. The fact that the convergence, determined from $\rho R,{ }^{4}$ is about the same for the SG3 and SG4 implosions suggests that the reduction in $R_{\text {burn }}$ for SG4 is largely a consequence of the smaller initial capsule radius (this is consistent with other measurements ${ }^{44}$ indicating that changing from SG3 to SG4 brought no significant improvement in overall implosion performance for capsules with $20-\mu \mathrm{m} \mathrm{CH}$ shells and 18-atm fill). The values of $R_{\text {xray }}$ show the same kind of variation with DPP type as the values of $R_{\text {burn, }}$ as shown in Fig. 5b. ${ }^{45}$ The 1-D $R_{\text {burn }}$ simulations (Fig. 5c, open triangles for SG3 and solid triangles for SG4) don't show as large a change with DPP type as the measured $R_{\text {burn }}$ or $R_{\text {xray }}$. An interesting question to address would be whether SG4 reduces the scatter in 3.6-atm capsule performance that was discussed in connection with Fig. 4, but comparative data are currently unavailable.

\section{Capsule shell thickness}

The shell thickness is known to have an effect on mix and convergence. ${ }^{4-9,10}$ Figure $6 \mathrm{a}$ shows the effect upon $R_{\text {burn }}$ (diamonds). The trend of these data is more fully revealed by averaging the data over capsules with similar shell thicknesses (Fig. 6b). As illustrated, $R_{\text {burn }}$ increases slowly as the shell thickness increases from 17 to $24 \mu \mathrm{m}$, with the values

and

$$
\begin{aligned}
& <R_{\text {burn }}(17 \mu \mathrm{m})>=29.1 \pm 0.4 \mu \mathrm{m}, \\
& <R_{\text {burn }}(20 \mu \mathrm{m})>=30.5 \pm 0.5 \mu \mathrm{m},
\end{aligned}
$$

$$
<R_{\text {burn }}(24 \mu \mathrm{m})>=32.8 \pm 1.1 \mu \mathrm{m} .
$$

The convergence for the thicker-shell capsules was slightly smaller, as reflected in the fact that the $\rho R$ values of those capsules are about the same (Fig. 6c) ${ }^{4}$ the larger burn radii for the thicker $24-\mu \mathrm{m}$ capsules reflect the smaller convergence. $R_{\text {xray }}$, also plotted in Fig. $6 \mathrm{~b}$, shows a similar trend. $R_{\text {burn }}$ for $1-\mathrm{D}$ simulations shows the same trend (triangles, Fig. $6 \mathrm{~d}$ ), but the absolute value, as remarked earlier, is significantly smaller.

\section{SUMMARY AND DISCUSSION}

In summary, we have described methods for measuring nuclear burn region sizes and presented the first measurements for a wide range of direct-drive implosion conditions, identifying systematic changes in burn region size due to changes in laser conditions and fuel 
capsule parameters. These measurements complement our related studies demonstrating systematic relationships between drive asymmetry, shell asymmetry, and burn asymmetry. ${ }^{11,12}$ Collectively, this work demonstrates the practicality and usefulness of emission imaging of nuclear burn, which directly reveals the spatial distributions of the fusion reactions that are the end result of all physical processes that affect capsule implosions.

Starting with laser drive conditions, it was shown that the burn radius in capsules with thin $(\sim 2 \mu \mathrm{m})$ glass shells and 18-atm fills varies strongly with total laser energy, going from $\sim 35 \mu \mathrm{m}$ at $6 \mathrm{~kJ}$ to $80 \mu \mathrm{m}$ at $23 \mathrm{~kJ}$ (all with 1-ns square pulses). Most measurements to date for capsules with plastic shells have been at $23 \mathrm{~kJ}$, so no conclusions were drawn here about energy variations (but future experiments may investigate this). Measurements also indicated that changing from the SG3 DPPs to the SG4 DPPs, for $20-\mu \mathrm{m} \mathrm{CH}$ shells and 18-atm fill, didn't significantly change shell convergence but did result in a somewhat smaller $R_{\text {burn }}$ that may simply reflect the small initial shell radius; this is consistent with other measurements ${ }^{44}$ indicating that changing from SG3 to SG4 brought no significant improvement in overall implosion performance for such capsules. Data for comparing $R_{\text {burn }}$ for SG3 and SG4 with 3.6-atm fill pressures are currently unavailable.

Looking next at capsule structure, it was seen that increasing the $\mathrm{CH}$ shell thickness from 17 to $24 \mu \mathrm{m}$ for 18 -atm fills resulted in the burn radius increasing from $30 \mu \mathrm{m}$ to $33 \mu \mathrm{m}$, a modest change largely attributed to the slightly smaller convergence of the more massive, thicker-shell capsules. Measurements have not yet been made for glass capsules with different shell thicknesses, but capsules with 2- $\mu \mathrm{m}$ glass shells have burn radii 2.5 times larger than capsules with $20-\mu \mathrm{m} \mathrm{CH}$ shells with equal laser energy $(23 \mathrm{~kJ})$ and fill pressure (18 atm). It was also demonstrated that reducing the $\mathrm{D}^{3} \mathrm{He}$ fill pressure from 18 to $3.6 \mathrm{~atm}$ in $20-\mu \mathrm{m} \mathrm{CH}$ shells resulted in little change in shell convergence but significant change in burn radius (from $31 \mu \mathrm{m}$ to $25 \mu \mathrm{m}$ ), a reduction largely attributed to increased fuel-shell mix for the more unstable 3.6-atm implosions. These data and interpretations are consistent with previous measurements of fuel $\rho R$ vs. gas pressure in implosions of DT-filled capsules, ${ }^{4}$ and we anticipate that more experiments and comparisons with simulations will be devoted to studying and quantifying the effects of mix.

The burn data were compared with x-ray images, which have a long history with ICF and therefore provide a very important point of comparison. As discussed in the text, x-ray images and burn images reflect different aspects of the compressed capsules and there is considerable ambiguity about how they should be compared and interpreted; the x-rays are most sensitive to $\mathrm{CH}$ from the inner part of the shell that is in contact with, or mixed into, the hot fuel. What was found is that the characteristic radius $R_{\text {xray }}$, calculated as described in ref. 14 and thought to be an indication of the inner boundary of hot $\mathrm{CH}$, is usually comparable to the characteristic burn radius $R_{\text {burn, }}$ which represents the median burn radius. There is, therefore, usually a radial overlap between the apparent burn region and the apparent inner $\mathrm{CH}$ location. This overlap may represent a region of atomic mix or a region where fingers of shell material extend into the fuel region, although interpretation of the x-ray images in the presence of mix is beyond the scope of this paper. A crucial fact about all of the data displayed in this article is that wherever $R_{\text {xray }}$ and $R_{\text {burn }}$ measurements are available for comparison (Figs. 4b, 5b, and 6b), they are comparable to each other and changes in one are tracked almost precisely by changes in the other. This is a strong independent confirmation that changes in burn region size measurements reflect true changes in the compressed capsule structure.

The burn data and x-ray data were both compared with predictions of 1-D simulations. It was found that while the 1-D burn radii were similar to the measured radii for capsules with 
thin glass shells (Fig. 3c), the predicted burn radii for capsules with $20-\mu \mathrm{m} \mathrm{CH}$ shells are smaller than the measured values by about $30 \%$ for 18 -atm fill and $50 \%$ for 3.6-atm fill (and the measured $R_{\text {xray }}$ was also larger than predicted for 18-atm fill). These discrepancies are qualitatively consistent with the discrepancies between predicted and measured values of areal density, which show that shell convergence is lower than predicted for all $\mathrm{CH}$-shell capsules studied. The dependence on fill pressure suggests that mix or instabilities could be a contributing factor. For a given amount of radial shell convergence, mix would be expected to make the burn region smaller by cooling the outer fuel regions, but we saw in Sec. IV-B that the convergence is very much smaller than predicted for lower fill pressures, probably because of mix; in addition, mix is known to truncate the burn in time, leading to a higherthan-predicted average radius during the burn interval. Another possible explanation for the simulation/measurement difference is preheat, which results in reduced compression due to increased pressure. For the glass-shell implosions, on the other hand, the roles of mix, hydrodynamic instabilities and preheat are expected to be substantially smaller at burn time in glass-shell implosions than in $\mathrm{CH}$-shell implosions ${ }^{40}$ and this could account for the closer agreement between simulation and data found in all such cases. In the future we hope to see if 2-D and 3-D simulations come closer to predicting the measured average shell convergence and measured burn region size. A thorough search for systematic errors that could lead to artificially broadened burn image data has been made, but no sources of error that could be large enough to account for the discrepancy in burn radii for $\mathrm{CH}$ shells have yet been identified. ${ }^{12,41}$

Comparisons of our $\mathrm{D}^{3} \mathrm{He}$ burn profiles with DT and DD burn profiles now being obtained by Disdier et al. at OMEGA with important new neutron imaging techniques, ${ }^{46}$ for hydrodynamically similar DT- and $\mathrm{D}_{2}$-filled capsules, are now being pursued and will be reported in the future. These comparisons could provide a test of consistency of the different burn-imaging methods and could potentially provide information about ion temperature profiles (through the local ratios of reaction rates).

\section{ACKNOWLEDGMENTS}

The authors express their gratitude to the OMEGA engineers and operations crew who supported these experiments. In addition, we would like to personally thank Michael Canavan, Candice Culligan, and Jocelyn Schaeffer for their continuous help. This work has been supported in part by LLE (Subcontract No. 412160-001G) and LLNL (Subcontract No. B543881), and by the U.S. Department of Energy Office of Inertial Confinement Fusion (Grant No. DE-FG03-03NA00058) and under Cooperative Agreement No. DE-FC5292SF19460, the University of Rochester, and New York State Energy Research and Development Authority. 


\section{APPENDIX: NOTES ON DETERMINING RADIAL BURN PROFILES FROM PENUMBRAL IMAGES}

As described in ref. 11, it is possible to determine the radial profile $S(r)$ of nuclear reactions in a burn region assumed spherically-symmetric by analyzing a penumbral image made using reaction products. The details of the approach used in this paper are slightly different from what is described in ref. 11, and we discuss them here along with alternative approaches and sample analyses. We start with the idealized assumption that the round imaging aperture has a perfectly defined, hard edge; a penumbral image of a point source would be uniform within a circular area and zero outside. This assumption is not warranted for imaging of DT burn with DT neutrons, but it was shown in ref. 12 that it should be sufficiently accurate for imaging of $\mathrm{D}^{3} \mathrm{He}$ burn with $\mathrm{D}^{3} \mathrm{He}$ protons. A few protons will scatter off the edge of the aperture, but their scattering angle is sufficiently large that they contribute only a small, relatively flat penumbral image background that disappears when the radial derivative is taken for analysis. In Sec. D, we discuss how this limitation can be removed either for neutron images or for small corrections with proton images if the effects of the aperture edge can be characterized.

\section{A. The problem}

The surface brightness of the spherically symmetric burn region is

$$
B(r)=\int_{-\infty}^{\infty} S\left(\sqrt{r^{2}+\ell^{2}}\right) d \ell .
$$

A penumbral image made with a hard-edged, round aperture of radius $R_{\mathrm{a}}$ is azimuthally symmetric with a radial profile $N(R)$ of detected protons per unit area, where $R$ is the radius measured with respect to the center of the image. The radial derivative of this image can be written

$$
\left.\frac{d N}{d R}\right|_{R=R_{d}+M x}=-\frac{1}{4 \pi M\left(L_{1}+L_{2}\right)^{2}} P_{c}(x)
$$

where

$$
P_{c}(x)=R_{d} \int_{-\pi}^{\pi} B\left(\sqrt{x^{2}+2 R_{c}\left(R_{c}+x\right)(1-\cos \theta)}\right) \cos \theta d \theta \quad .
$$

In Eq. 11, $L_{1}$ and $L_{2}$ are the source-aperture and aperture-detector distances, $M=L_{2} / L_{1}$ is the geometric magnification of the penumbral camera, and $R_{\mathrm{d}} \equiv(M+1) R_{\mathrm{a}}$ is the radius of the aperture image at the detector. In Eq. $12, R_{\mathrm{c}} \equiv R_{\mathrm{a}}(M+1) / M$ is the radius of the aperture's projection at the location of the burn region, as seen from the detector, and the angle $\theta$ is measured relative to the center of this projection. As discussed in ref. $11, P_{c}(x)$ is a set of integrals through the surface brightness of the burn region along parallel paths that are curved but become straight in the limit $R_{\text {burn }} / R_{\mathrm{c}}<<1$ :

$$
P_{c}(x) \stackrel{R_{\text {burn }} / R_{a} \rightarrow 0}{\longrightarrow} P(x)=\int_{-\infty}^{\infty} B\left(\sqrt{x^{2}+\ell^{2}}\right) d \ell \quad .
$$

$P_{c}(x)$ can be thought of as a one-dimensional projection of the surface brightness $B(r)$.

$P_{c}(x)$ can be obtained experimentally from $\mathrm{d} N / \mathrm{d} R$ through Eq. 11, and if $R_{\text {burn }} / R_{\mathrm{c}}<<1$ it can be used to obtain $B(r)$ through Abel inversion. Similarly, $B(r)$ can be Abel inverted to obtain the source profile $S(r)$. If $R_{\text {burn }} / R_{\mathrm{c}}$ is not negligible, then the straight-line-integral $P(x)$ can be calculated approximately from $P_{c}(x)$ before the Abel inversion process: ${ }^{47}$

$$
P(x) \cong \sqrt{1+x / R_{c}} P_{c}(x)=-\left.4 \pi M\left(L_{1}+L_{2}\right)^{2} \sqrt{\frac{R}{R_{d}}} \frac{d N}{d R}\right|_{R=R_{d}+M x} .
$$


A simple analytic solution for direct calculation of the double Abel inversion relating $S(r)$ to $P(x)$ was described in ref. 48 in connection with a different application (imaging of a spherically symmetric source with a linear slit aperture):

$$
S(r)=-\left.\frac{1}{2 \pi r} \frac{d P(x)}{d x}\right|_{x=r} \quad .
$$

Eq. 15 works well for perfect data (no noise or other distortions, infinitesimal sampling width, and $R_{\mathrm{burn}} / R_{\mathrm{c}}<<1$ ), but it isn't ideal for the data discussed in this paper, even though the data generally satisfy the condition $R_{\text {burn }} / R_{\mathrm{c}}<<1$, for several reasons. First, the statistics generally aren't good enough even after the data are binned (see the caption of Fig. 1a) except for the high-yield glass-shell capsules. Second, it becomes inaccurate when the data are binned, since this is equivalent to imposing a finite sampling width and results in artificial broadening of the inferred $S(r)$ and smoothing of any feature that is not much larger than the sampling width. ${ }^{49}$ Finally, it has a problem at $r=0$ where, even if $P(0) \equiv 0$, any noise or measurement uncertainty in the data near $r=0$ translates into uncertainties in $S$ that become infinite as $r \rightarrow 0$ (this is not a defect in Eq. 15, but a consequence of the fact that the central emissivity value applies to a small volume and has very little effect on line integrals through the surface brightness). Nevertheless, the direct method works away from $r=0$ if statistics allow, and application to the current data are illustrated in Sec. C, below.

\section{B. Inferring $S(r)$ from least-squares fits}

As with many Abel-inversion applications, the statistics issue can be improved by fitting the raw data with analytic functions that automatically smooth out some of the statistical fluctuations in $P(x)$ and simultaneously enforce reasonable behavior at the singular point $\mathrm{r}=0$. In ref. 11, we proposed the use of powers of parabolas to represent $S(r)$ because these map analytically to other powers of parabolas for $P(x)$; the experimental $P(x)$ can be fit to powers of a parabola and $S(r)$ found analytically. This makes possible a range of profile shapes for $S(r)$ varying from hollow to peaked, with the limiting peaked shape being a Gaussian when the power goes to infinity, and works well because many of the data sets analyzed are statistically consistent with a Gaussian shape for $S(r)$. However, with many data sets there were indications that slightly better fits might be achieved with a profile more peaked than Gaussian, so here we take a different approach that has several advantages.

Instead of using a set of functions to fit to $P(x)$ and analytically deducing $S(r)$ using Eq. 15, we start by representing $S(r)$ by the family of super- and sub-Gaussians

$$
S(r)=S_{0} e^{-\left(r / r_{0}\right)^{2 / p}}
$$

where $r_{0}$ is the " $1 / \mathrm{e}$ " radius and $p$ is the "peakedness" of the emission profile ( $p=0$ is flat, $p=1$ is Gaussian, $p>1$ is more centrally peaked than Gaussian). From a given trial function $S(r)$, the functions $B(r), P_{c}(x)$ and $\mathrm{d} N / \mathrm{d} R$ can be calculated numerically from Eqs. 10,12 and 11 . For comparison with experimental data, the predicted $\mathrm{d} N / \mathrm{d} R$ must then be convolved with a boxcar function to model the actual binning used in tabulating measured values. To determine the most probable profile $S(r)$, the parameters $R_{\mathrm{d}}, S_{0}, p$, and $r_{0}$ are varied and the values leading to a minimum $\chi^{2}$ fit of predicted to measured data are determined along with their statistical uncertainties. ${ }^{50}$ The deduced $S(r)$ can then be described by $S_{0}$ (or by the total yield), $p$, and $r_{0}$, though as shown below it turns out to more useful to parameterize the radial size by the mean yield-weighted burn radius $\langle r\rangle_{S}=\int r S(r) r^{2} d r / \int S(r) r^{2} d r$ or by the median burn radius $r_{\mathrm{S} \text {,median }}$ containing half the yield rather than by $r_{0}$. 
Figure 7 illustrates sample shapes of $S(r)$ together with corresponding functions $B(r)$ and $P(x)$. We see immediately that small differences in the shape of $P(x)$ translate into much larger differences in the shape of $S(r)$, which is characteristic of inversion procedures. This means that finite errors in the raw data are amplified into much larger errors in the shape of $S(r)$, particularly for small $r$, though it will turn out that errors in the characteristic radius are not amplified by the inversion process.

Figure 8 illustrates what happens when this technique is applied to the measured $\mathrm{d} N / \mathrm{d} R$ data shown in Figs. 1a and 1e. Figures $8 \mathrm{a}$ and $8 \mathrm{~d}$ show contour plots of total $\chi^{2}$ vs. $p$ and $r_{0}$ (using at every point the values of $R_{\mathrm{c}}$ and $S_{0}$ that minimize $\chi^{2}$ ). In each case there is a well-defined location corresponding to the best fit (lowest $\chi^{2}$ ). Figures $8 \mathrm{~b}$ and $8 \mathrm{e}$ show the same contours parameterized by $\langle r\rangle_{S}$ rather than $r_{0}$, and Figs. 8c and 8f show parameterization by $r_{S}$, median. Notice that either $\langle r\rangle_{S}$ or $r_{S}$, median is much more independent of the peakedness parameter $p$ than $r_{0}$; the same conclusion has been reached using a wide range of functional forms for $S(r)$ and a wide range of data sets. The problem with $r_{0}$ is that a change in the central value of $S(r)$ changes the $1 / \mathrm{e}$ radius but has little effect on $P(x)$. In general, $r_{S, \text { median }}$ seems slightly better than $\langle r\rangle_{S}$, so from now on we will define the characteristic burn radius $R_{\text {burn }}$ to be $r_{S \text {, median. Notice also that the percent statistical }}$ uncertainty in $p$ is much larger than the uncertainty in $R_{\text {burn }}$. This is because errors in radial size do not get amplified in the inversion process, as can be shown analytically using Eq. 15 for the case $R_{\text {burn }} / R_{\mathrm{a}}<<1$ : the mean burn radius $\left\langle r>_{\mathrm{S}}\right.$ is always exactly twice the average radius of $P(x):\langle r\rangle_{P} \equiv \int x P(x) d x / \int P(x) d x=0.5\langle r\rangle_{S}$, regardless of the shape of $S(r)$. This means that a fractional error in $\langle r\rangle_{\mathrm{p}}$ results in the same fractional error in $\left\langle_{r}\right\rangle_{\mathrm{s}}$. A similar result holds for the median radius $\left(r_{P, \text { median }}=0.44 r_{S \text {, median }}\right)$.

Figures $1 \mathrm{c}, 1 \mathrm{~g}, 1 \mathrm{~d}$ and $1 \mathrm{~h}$ show the radial profiles of $S(r)$ and $B(r)$ corresponding to the same data and fits, together with uncertainties deduced from the fitting procedure. The largest uncertainties in $S(r)$ and $B(r)$ are at the center, and the uncertainties get larger with each level of inversion. The large uncertainties at $r=0$ simply reflect the fact that changes in the central emissivity have very little effect on penumbral images.

The family of functions represented by Eq. 16 doesn't extend to hollow profiles, but can easily be extended in that direction through use of different functions. In addition, more complicated radial profiles using series expansions (e.g., of Chebyshev polynomials) can be used if statistics allow. But if yields are high enough, the direct calculation of $S(r)$ becomes practical.

\section{Direct calculation of $S(r)$}

Subject to the conditions discussed in Sec. A, Eq. 15 allows direct calculation of $S(r)$ from $P(x)$ calculated with Eq. 14. Figure 9 shows how this works out for the two implosions analyzed above. This approach requires knowledge of $R_{\mathrm{d}}$; for the calculations illustrated in Fig. 9 the values of $R_{\mathrm{d}}$ inferred from the fitting method were used. In each case the binning width was made as small as possible consistent with counting statistics. Shot 27456 has a high enough proton yield $\left(\sim 2 \times 10^{10}\right)$ to make this method work for $r \geq R_{\text {burn }} / 3$; as $r$ is reduced the calculated values start to fall because of the effects of binning near the central peak, and as $r$ approaches 0 the result is completely unreliable because of the singularity in Eq. 15 . The yield is much lower for shot $37156\left(\sim 4 \times 10^{8}\right)$, so the statistical errors on the calculated values of $S(r)$ are much larger and the shape of $S(r)$ is somewhat ill defined. The calculated value near $\mathrm{r}=0$ happened to be about right, but this was partly a matter of luck; changing the binning resulted in erratic values. 


\section{Generalization to apertures without "hard edges" and neutron imaging}

If a penumbral-imaging aperture has a perfect, opaque edge, then the radial derivative $\mathrm{d} N / \mathrm{d} R$ of a penumbral image of a point source will be a delta function. If not, and if $\mathrm{d} N / \mathrm{d} R$ for a point source can be either calculated or measured, then it can be incorporated directly into the method described in Sec. B. Before comparison with measured values of $\mathrm{d} N / \mathrm{d} R$, each predicted function calculated from a trial function $S(r)$ through Eqs. 10,12 and 11 need only be convolved by an appropriate smearing function $F(R)$ before being convolved by the boxcar function that models the data binning. $F\left(R^{\prime}\right)$ is simply $\mathrm{d} N / \mathrm{d} R$ for a point source evaluated at $R=R_{\mathrm{d}}+R^{\prime}$ and normalized to have unit integral. If the direct-calculation method is to be used instead of the least-squares fitting method, then the data must be deconvolved to remove the effect of $F$. 
References

1. J. Nuckolls, L. Wood, A. Theissen et al., Nature (London) 239, 139 (1972).

2. J. D. Lindl, Inertial Confinement Fusion: The Quest for Ignition and Energy Gain Using Indirect Drive (Springer-Verlag, New York, 1998).

3. S. Atzeni and J. Meyer-Ter-Vehn (2004). The Physics of Inertial Fusion. New York, NY: Oxford University Press Inc.

4. C. K. Li, F. H. Séguin, J. A. Frenje et al., Phys. Rev. Lett. 89, 165002 (2002).

5. D. D. Meyerhofer, J. A. Delettrez, R. Epstein et al., Phys.Plasmas 8, 2251 (2001).

6. R. B. Radha, J. Delettrez, R. Epstein et al., Phys. Plasmas 9, 2208 (2002).

7. S. P. Regan, J. Delettrez, F. J. Marshall et al., Phys. Rev. Lett. 89, 085003 (2002).

8. C. R. Christensen, D. C. Wilson, C. W. Barnes et al., Phys. Plasmas 11, 2771 (2004).

9. D. C. Wilson, C. W. Cranfill, C. R. Christensen et al., Phys Plasmas 11, 2723 (2004).

10. P. B. Radha, V. N Goncharov, T. J. B. Collins et al., Phys. Plasmas 12, 032702 (2005).

11. F. H. Séguin, J. L. DeCiantis, J. A. Frenje et al., Rev. Sci. Instrum. 75, 3520 (2004).

12. J. L. DeCiantis, F. H. Séguin, J. A. Frenje et al., Rev. Sci. Instrum. 77, 043503 (2006).

13. F. H. Séguin, J. L. DeCiantis, J.A. Frenje et al., Bull. Am. Phys. Soc. 49, 63 (2004); F. H. Séguin, C.K. Li, J. L. DeCiantis et al., Bull. Am. Phys. Soc. 50, 114 (2005); and "Measured Effects of drive asymmetry and shell asymmetry on nuclear burn region symmetry in direct-drive ICF implosions" (to be submitted Phys. Plasmas).

14. S. P. Regan, J. A. Delettrez, R. Epstein et al., Phys. Plasmas 9, 1357 (2002).

15. D. Ress, L. B. DaSilva, R. A. London et al., Rev. Sci. Instrum. 66, 579 (1995).

16. V. A. Smalyuk, T. R. Boehly, L. S. Iwan et al., Rev. Sci. Instrum. 72, 635 (2001).

17. F. J. Marshall, J. A. Delettrez, R. Epstein et al., Phys. Plasmas 7, 2108 (2000).

18. F. H. Séguin, C. K. Li, J. A Frenje et al., Phys. Plasmas 9, 3558 (2002).

19. F. H. Séguin, J. A. Frenje, C. K. Li et al., Rev. Sci. Instrum. 74, 975 (2003).

20. F. H. Séguin, C. K. Li, J. A. Frenje et al., Phys. Plasmas 9, 2527 (2002).

21. C. K. Li, F. H. Séguin, J. A. Frenje et al., Phys. Plasmas 10, 1919 (2003).

22. R. D. Petrasso, J. A. Frenje, C. K. Li et al., Phys. Rev. Lett. 90, 095002 (2003).

23. C. K. Li, D. G. Hicks, F. H. Séguin et al., Phys. Plasmas 7, 2578 (2000).

24. M. C. Richardson, P. W. McKenty, F. J. Marshall et al., in Laser Interaction and Related Plasma Phenomena, edited by H. Hora and G. H. Miley (Plenum Press, New York, 1986), Vol. 7, pp. 421-448

25. J. Delettrez, R. Epstein, R. C. Richardson et al., Phys. Rev. A 36, 3926 (1987).

26. K. A. Nugent and B. Luther-Davies, J. Appl. Phys. 58, 2508 (1985).

27. D. Ress, R. A. Lerche, R. J. Ellis et al., Rev. Sci. Instrum. 59, 1694 (1988).

28. R. A. Lerche, D. Ress, R. J. Ellis et al., Laser Part. Beams 9, 99 (1991).

29. L. Disdier, R. A. Lerche, J. L. Bourgade et al., Rev, Sci. Instrum. 75, 2134 (2004).

30. Y. -W. Chen, M. Yamanaka, N. Miyanaga et al., Opt. Commun. 73, 337 (1989).

31. M. Nakai, M. Yamanaka, H. Azechi et al., Rev. Sci. Instrum. 61, 3235 (1990).

32. A. P. Fews, P. A. Norreys, F. N. Beg et al., Phys. Rev. Lett. 73, 1801 (1994).

33. T. R. Boehly, D. L. Brown, R. S. Craxton et al., Opt. Commun. 133, 495 (1997).

34. Y. Lin, T. J. Kessler, and G. N. Lawrence, Opt. Lett. 20, 764 (1995).

35. S. Skupsky, R. W. Short, T. Kessler et al., J. Appl. Phys. 66, 3456 (1989).

36. S. P. Regan, J.A. Marozas, R.S. Craxton et al., J. Opt. Soc. Am. B 22, 998 (2005).

37. T. R. Boehly, V. A. Smalyuk, D. D. Meyerhofer et al., J. Appl. Phys. 85, 3444 (1999).

38. R. A. Forties and F. J. Marshall, Rev. Sci. Instrum. Vol. 76, 073505 (2005).

39. C. K. Li, F. H. Séguin, D. G. Hicks et al., Phys. Plasmas 8, 4902 (2001). 
40. M. D. Rosen and J. H Nuckolls, Phys. Fluids 22, 1393 (1979).

41. Calculations showed insignificant image broadening associated with proton scattering in the capsule, aperture, and detector filters, while experiments showed no indication of different results with different aperture size or magnification. Neither experiments nor simulations revealed any serious field effects. ${ }^{12}$

42. V. Y. Glebov, C. Stoeckl, T. C. Sangster et al., Rev. Sci. Instrum. 75, 3559 (2004).

43. The plotted $R_{\text {burn }}$ and $<\rho R>$ results for individual implosions already represent a mean value of the measurements obtained from that implosion; there were as many as six measurements of $\mathrm{R}_{\text {burn }}{ }^{12}$ and five of $\rho \mathrm{R}$ for each implosion. When the mean value is calculated for an implosion type, all of these measurements are weighted equally.

44. S. P. Regan, J.A. Delettrez, V. Yu. Glebov et al., Bull. Am. Phys. Soc. 49, 62 (2004).

45. The $S G 3 R_{x r a y}$ data point was taken from a hydrodynamically-equivalent $15 \mathrm{~atm} \mathrm{D}_{2}$ gas fill implosion (shot 22546). ${ }^{7}$ The substitution is reasonable; similar $\mathrm{R}_{\mathrm{xray}}$ results are obtained from $\mathrm{D}_{2}$ and $\mathrm{D}^{3} \mathrm{He}$ gas implosions [J. L. DeCiantis, MS Thesis, Massachusetts Institute of Technology (2005)].

46. L. Disdier, A. Rouyer, I. Lantuéjoul et al., "Inertial confinement fusion neutron images", to be published in Phys. Plasmas (2006).

47. The correction factor $\sqrt{1+x / R_{c}}=\sqrt{R / R_{d}}$ in Eq. 14 can be found by calculating the integrals for a circular disk with uniform surface brightness and keeping the lowestorder terms. Since any radial profile of surface brightness can be made up from a superposition of disks, the result is independent of radial profile. This is a better correction than that shown in Eq. 7 of ref. 11, which was found empirically. In any case, the correction is too small to be important for the data considered here.

48. C. M. Vest and D. G. Steel, Opt. Lett. 3, 54 (1978).

49. M. M. Mueller, Opt. Lett. 4, 351 (1979).

50. The parameter $R_{\mathrm{d}}$ is the radius on the detector corresponding to the center of the penumbra. In principle it is known in advance from the dimensions of the imaging system, but it must be known very accurately and including it as a fit parameter allows for the possibility of small errors in camera positioning. Two more parameters that need to be known are the coordinates of the center of the penumbral image. This is found by doing a simplified version of the analysis described in the text, but varying the assumed center coordinates and finding the values that give the smallest burn radius (since any displacement from the true center broadens the average penumbra and the inferred burn radius). 
Shot 27456
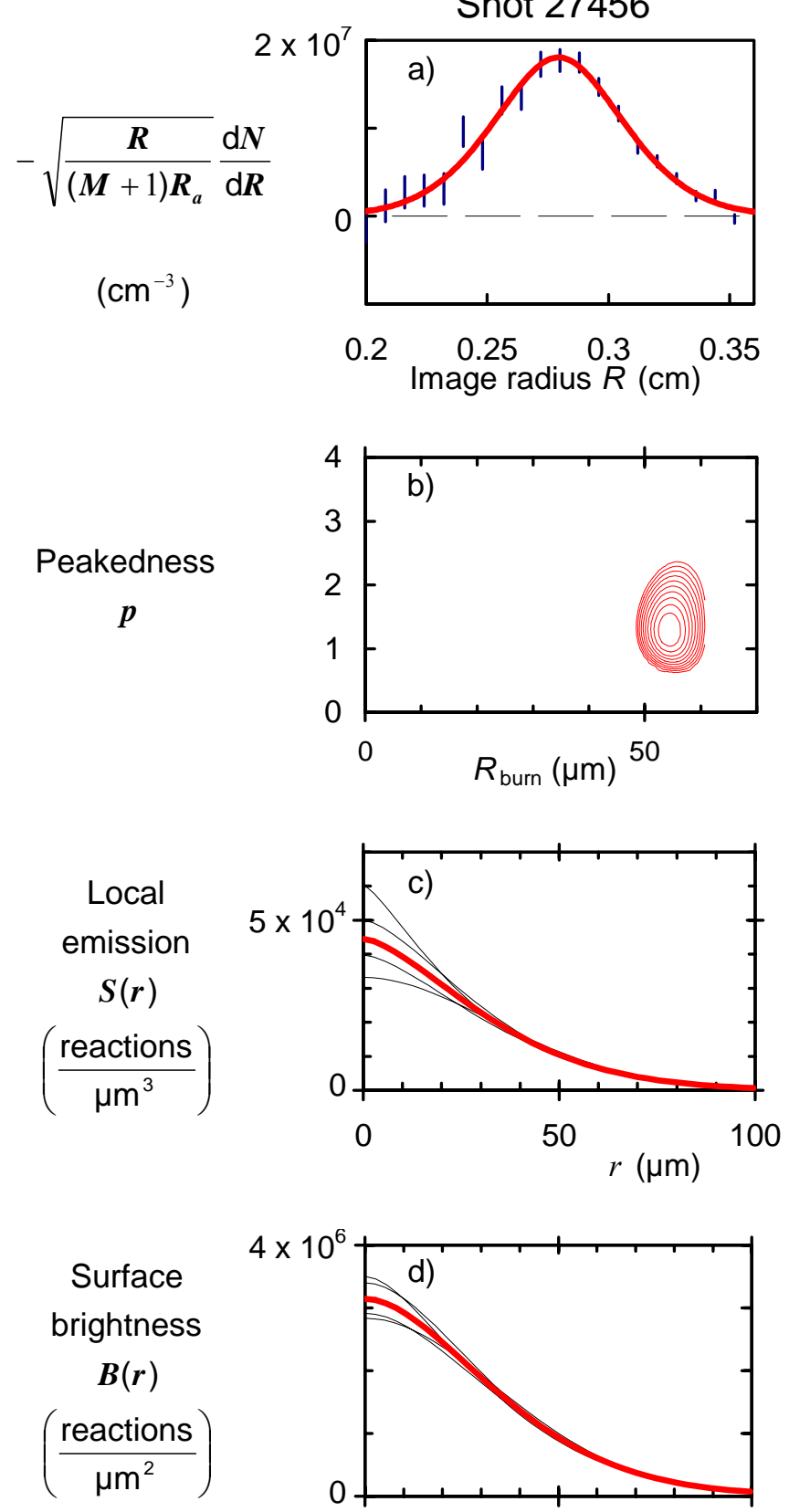
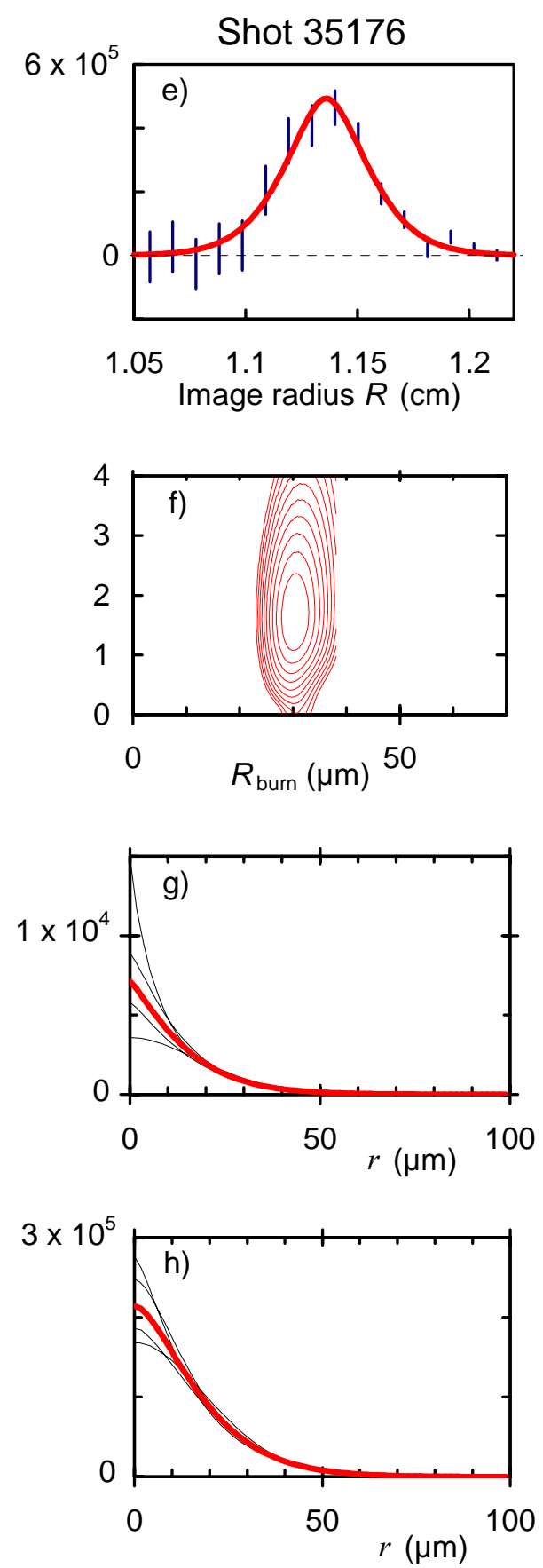

FIG. 1. (Color online) Data illustrating burn-region analysis of two very different implosions at OMEGA. The left-hand column corresponds to shot 27456 (2- $\mu \mathrm{m}$-thick, glass-shell capsule), while the right-hand column corresponds to shot 35176

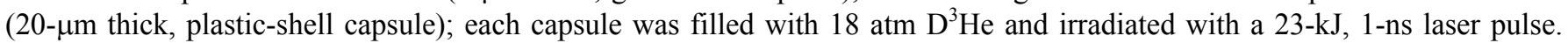
The vertical lines in (a) and (e) represent statistical error bars for measured values of $\mathrm{d} N / \mathrm{d} R$; the locations of all individual proton tracks on the penumbral image detector are measured to a fraction of a micron, but $N(R)$ needs to be binned with a finite interval in $R$ in order to achieve acceptable statistics. Note that the significance of the square root appearing as a coefficient of $\mathrm{d} N / \mathrm{d} R$ in the vertical axis labels is discussed in the Appendix in connection with Eq. (14); this slowly varying coefficient is very close to 1.0 for the data shown here. The heavy lines in (a) and (e) are best fits to the data using the approach described in Sec. III and in the Appendix. Contour plots showing total $\chi^{2}$ as a function of $R_{\text {burn }}$ and peakedness $p$ are shown in (b) and (f); the contour levels correspond to $\chi_{\text {minimum }}^{2}+1, \chi_{\text {minimum }}^{2}+2, \ldots$ In (c) and (g) the inferred radial distributions $S(r)$ of $\mathrm{D}^{3} \mathrm{He}$ reactions in the burn regions are shown; each heavy line corresponds to a best fit, while the lighter lines show alternate fits resulting in the total $\chi^{2}$ being larger than the minimum value by 1 (indicating an approximate error envelope for the best-fit profile). The corresponding surface brightness distributions $B(r)$ are shown in (d) and (h). The parameters describing the profiles $S(r)$ are $R_{\text {burn }}=54 \pm 2 \mu \mathrm{m}$ and $p=1.35 \pm 0.25$ (shot 27456) and $R_{\text {burn }}=29 \pm 2.5 \mu \mathrm{m}$ and $p=1.7 \pm 0.6$ (shot 35176). 


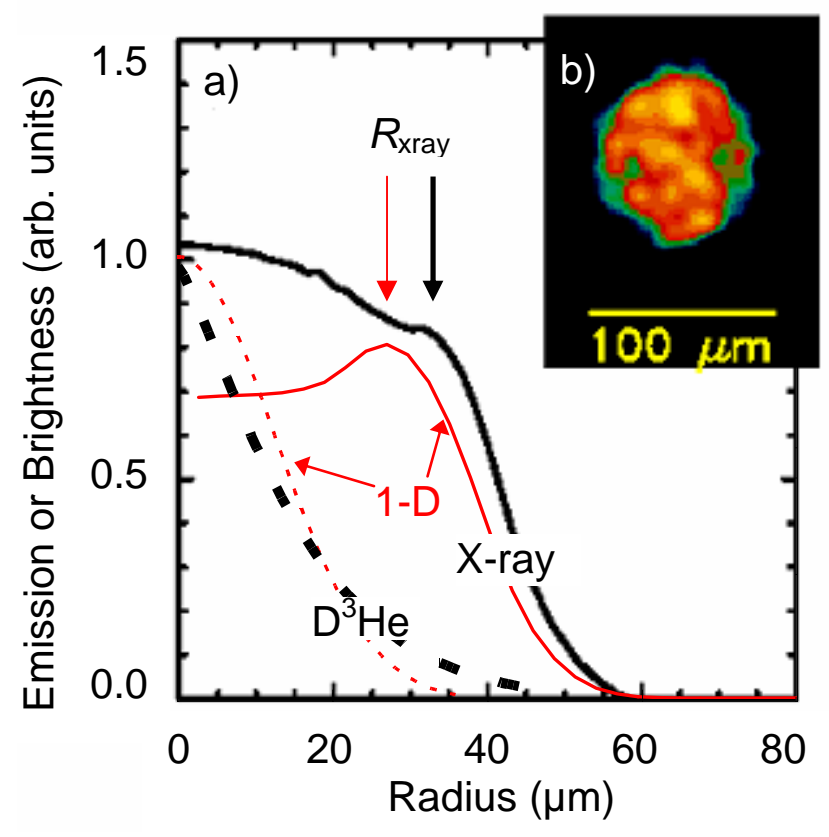

FIG. 2. (Color) Comparison of the measured local $\mathrm{D}^{3} \mathrm{He}$ emission profile, the measured $\mathrm{x}$-ray surface-brightness profile, and 1-D simulations for shot 35176. A $4-5 \mathrm{keV} x$-ray image taken at peak nuclear burn time (a 40-ps exposure) is shown in (b), and its radial profile is shown in (a) together with a 1-D simulation. The $\mathrm{D}^{3} \mathrm{He}$ burn profile (from Fig. 1g) is also shown in (a), together with a 1-D simulation. In all cases the measured profiles are bold and the 1-D profiles are fine. The $\mathrm{D}^{3} \mathrm{He}$ profiles are arbitrarily normalized to have the same value at $r=0$. 

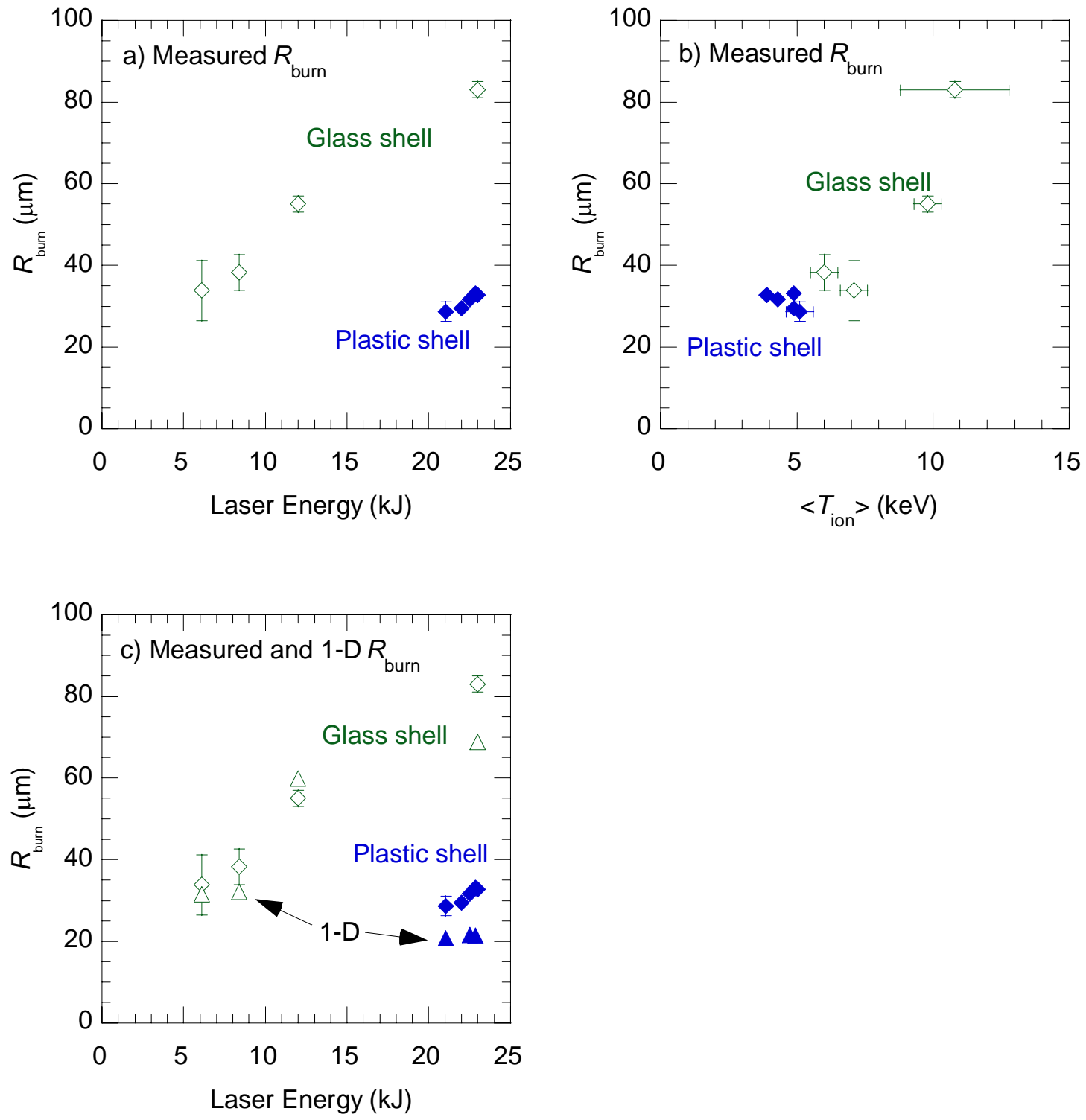

FIG. 3. (Color online) (a) Data showing the relationship of burn region size to laser energy for implosions of capsules with 18 -atm $\mathrm{D}^{3} \mathrm{He}$ fill and either $20-\mu \mathrm{m}$-plastic or $2-\mu \mathrm{m}$-glass shells. For the thin-glass-shell, exploding-pusher implosions (open diamonds), increasing the laser energy results in a dramatically larger $\mathrm{D}^{3} \mathrm{He}$ burn region radius. For the thick- $\mathrm{CH}$-shell, compressive implosions, $R_{\text {burn }} \approx 30 \mu \mathrm{m}$ for $23 \mathrm{~kJ}$ laser energy (solid diamonds), but data are not currently available for lower laser energies. The ion temperature was strongly correlated with $R_{\text {burn }}$, as shown in (b) where $R_{\text {burn }}$ has been plotted versus the burn-averaged ion temperature $<\mathrm{T}_{\text {ion }}>$ obtained with neutron time-of-flight systems. (c) Comparison of $R_{\text {burn }}$ measurements with values from 1-D simulations (triangles). 

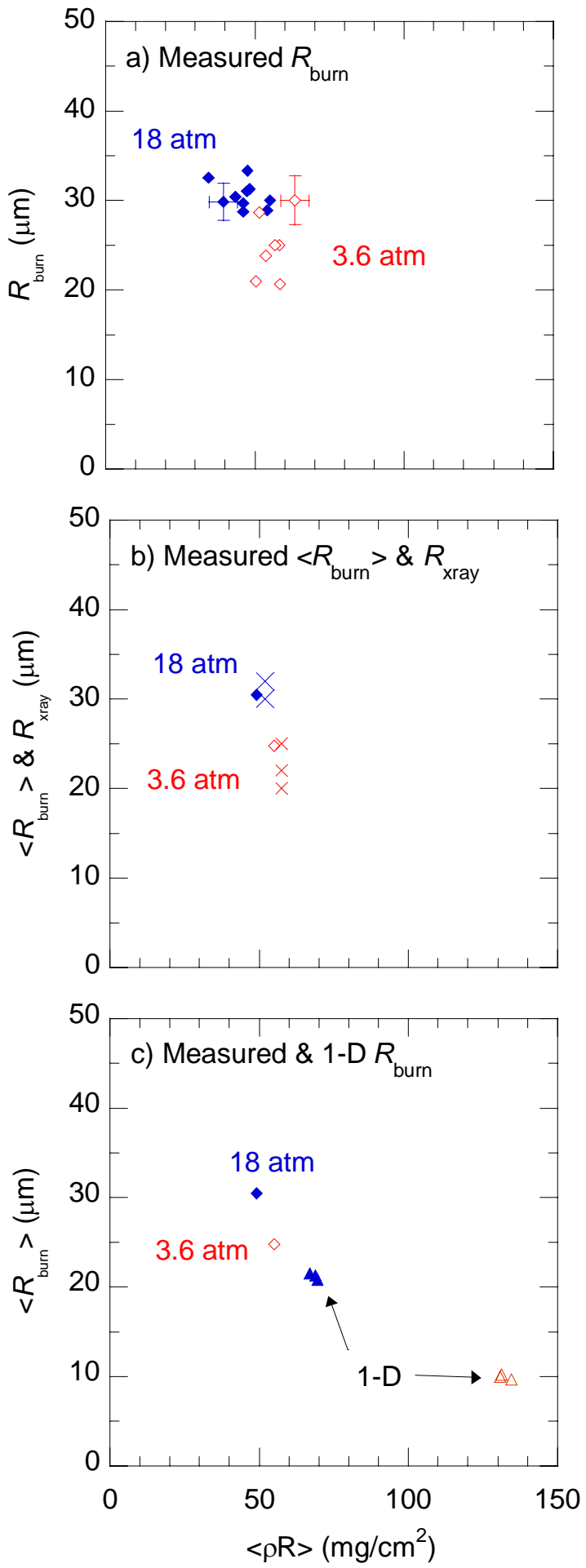

FIG. 4. (Color online) (a) A significant difference in the $\mathrm{D}^{3} \mathrm{He}$ burn size is shown for 18-atm (closed diamonds) and 3.6-atm (open diamonds) fill pressures in implosions of $\mathrm{D}^{3} \mathrm{He}$-filled capsules with 19 - $20 \mu \mathrm{m}$ plastic-shells. $R_{\text {burn }}$ is plotted as a function of the areal density $<\rho R>$ measured from proton energy downshifts. (b) The averages of data in (a) are displayed with fuel-shell interface estimates $\left(R_{\text {xray }}\right)$ for 18-atm (large $\left.\mathrm{X}\right)$ and 3.6-atm (small $\mathrm{x}$ ) implosions, demonstrating agreement in the trends of both $R_{\text {burn }}$ and $R_{\text {xray. }}$ (c) When the average data are displayed with 1-D calculated $R_{\text {burn }}$ (triangles), the same trend is present, but the simulations predict lower $R_{\text {burn }}$ values overall and much larger values of. $\rho R$ with decreased fill pressure. 

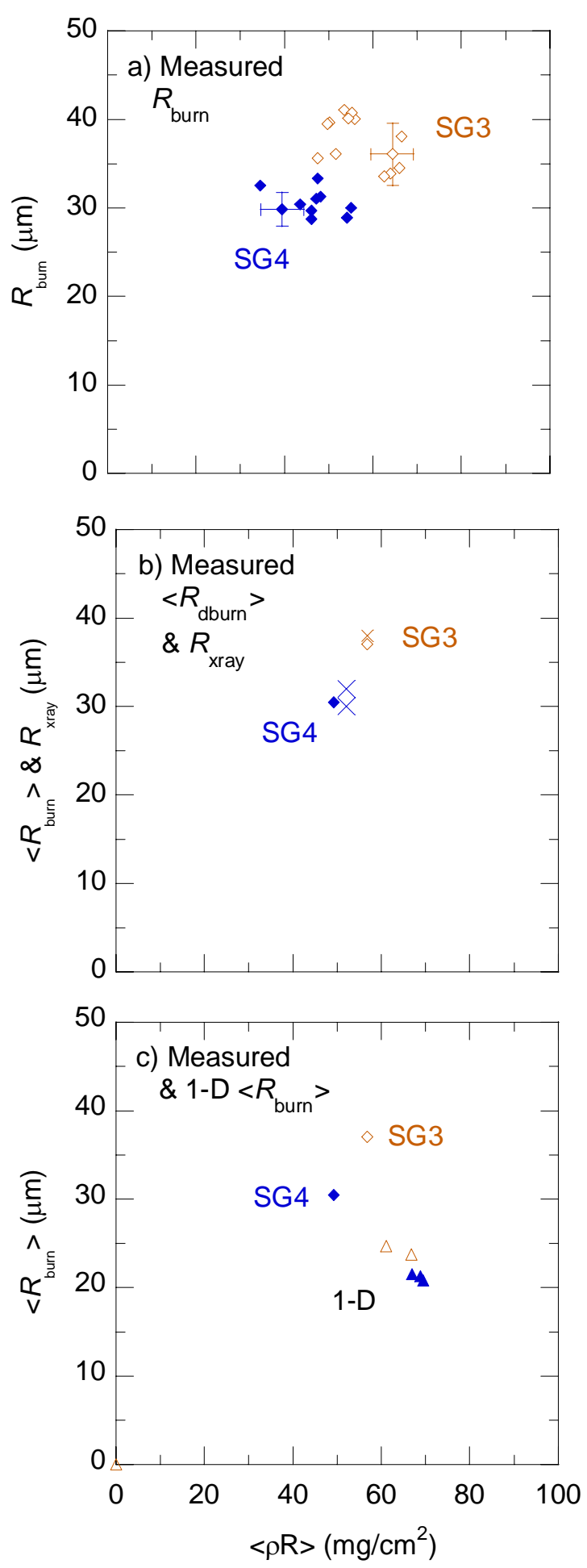

FIG. 5. (Color online) (a) Consistently smaller burn radii are produced with SG4 targets and phase plates (solid diamonds) than with SG3 (open diamonds). $R_{\text {burn }}$ is plotted as a function of the areal density $\rho R$ for implosions of capsules with $19-20 \mu \mathrm{m}$ thick plastic shells and 18 atm $\mathrm{D}^{3} \mathrm{He}$ fill. (b) The averages of burn radii burn data in (a) are displayed with fuel-shell interface estimates $\left(R_{\text {xray }}\right)$ from $\mathrm{SG} 3$ (small $\mathrm{x}$ ) and SG4 (large $\mathrm{X}$ ) implosions, ${ }^{44}$ demonstrating agreement in the trends of $R_{\text {burn }}$ and $R_{\text {xray }}$. (c) When the average $R_{\text {burn }}$ data are displayed with the 1-D values (triangles), the same trend is present, but the simulations predict lower $R_{\text {burn }}$ values and a smaller change in $R_{\text {burn }}$ with the change in phase plates. The reduction in $R_{\text {burn }}$ with the change from SG3 to SG4 seems largely dominated by the reduction in initial capsule radius from $\sim 470$ to $430 \mu \mathrm{m}$. 

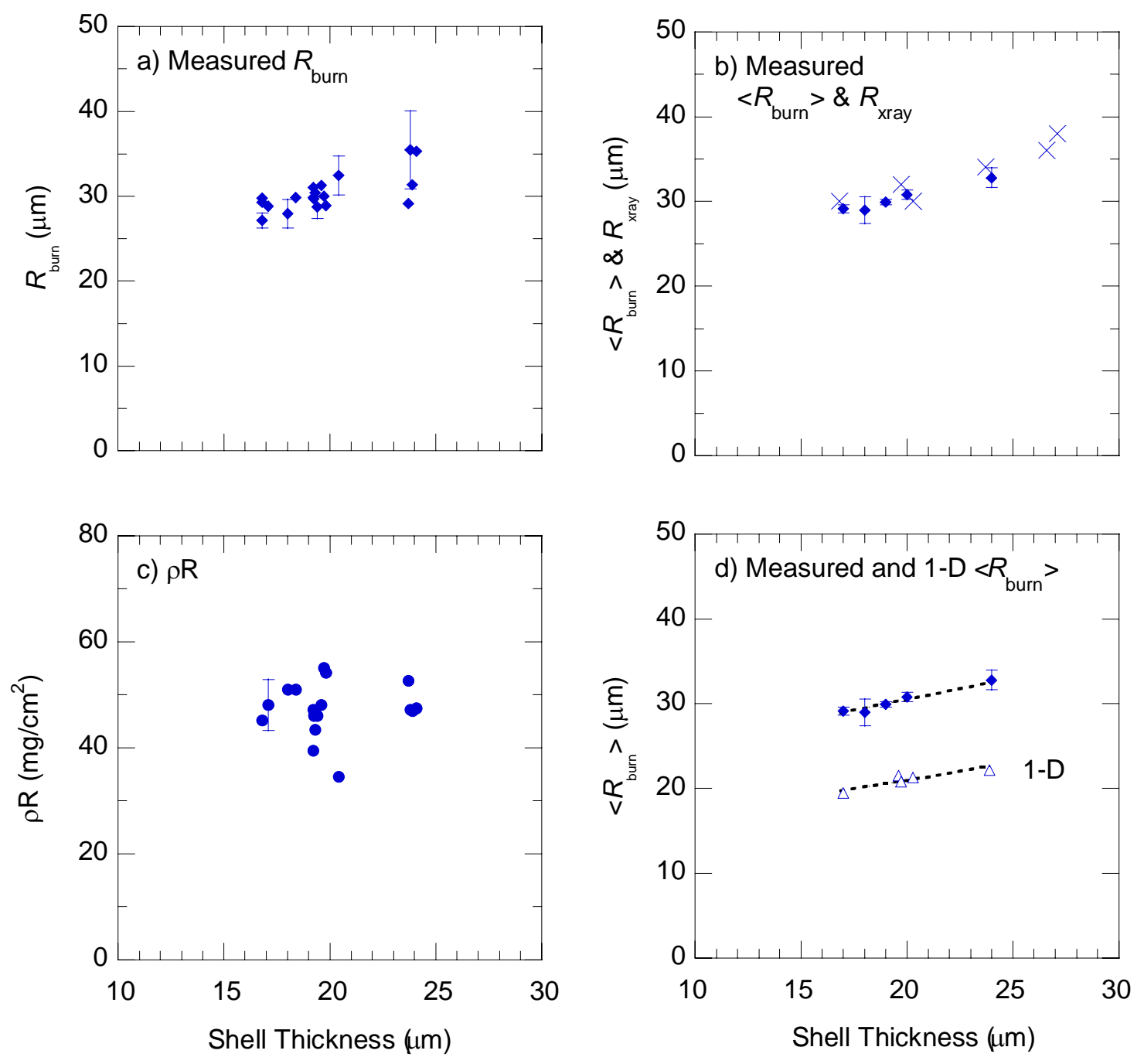

FIG. 6. (Color online) (a) The dependence of $R_{\text {burn }}$ on plastic shell thickness provides information about mix and convergence ${ }^{2-10}$. (b) The trend is more obvious when the $R_{\text {burn }}$ data for similar capsule thicknesses are averaged (diamonds). The fuel-shell interface estimates $\left(R_{\text {xray }}, \mathrm{X}\right)$ for these implosions and others demonstrate virtually the same trend. (c) The areal densities $\rho \mathrm{R}$ measured for the same implosions were only weakly dependent on shell thickness. (d) Predicted values of $R_{\text {burn }}$ from 1-D simulations (triangles) show the same trend as the measurements but lower values. 

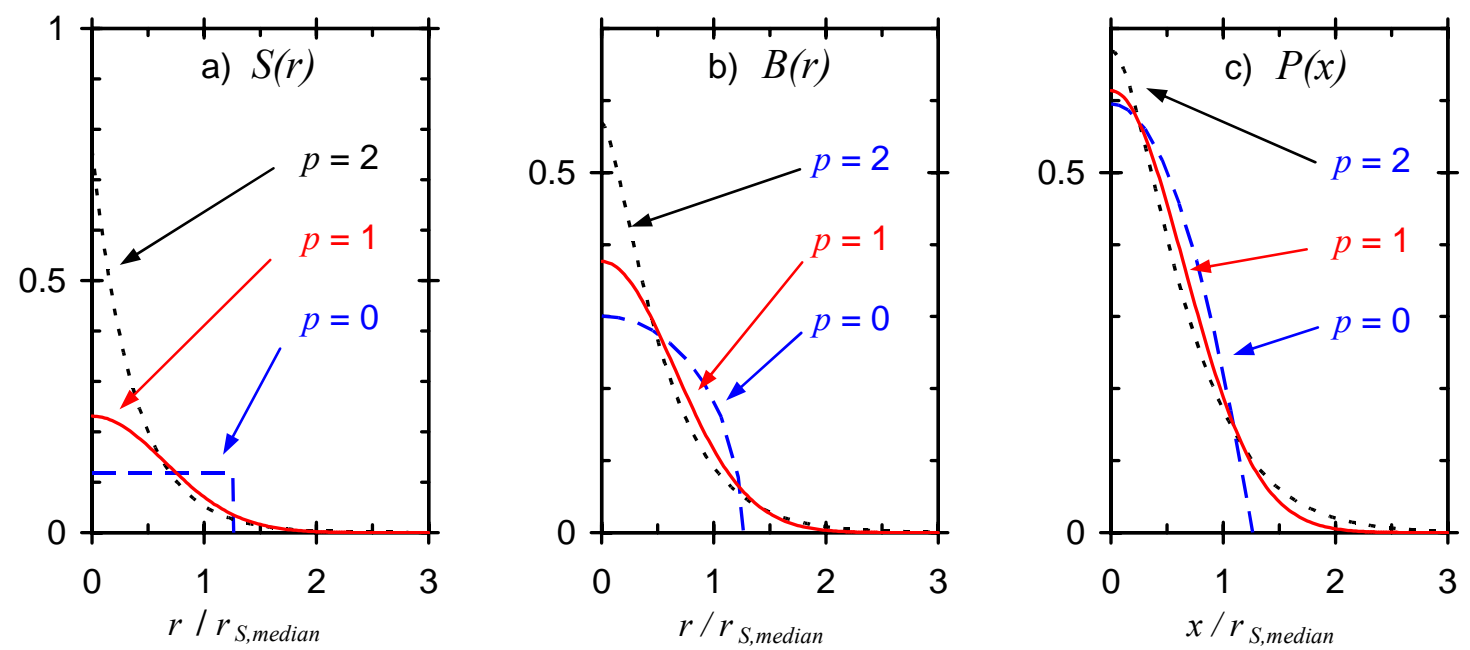

FIG. 7. (Color online) (a) $S(r)$ from Eq. 11 for $p=0$, 1, and 2, normalized so each curve has the same total yield. As discussed in the text, $r_{\mathrm{s} \text {,median }}$ is the median radius (containing half the yield). (b) The corresponding curve for $B(r)$. (c) $P(x)$ for the case of no data binning. Note that hollow profiles of $S(r)$ can easily be added to this family of functions.
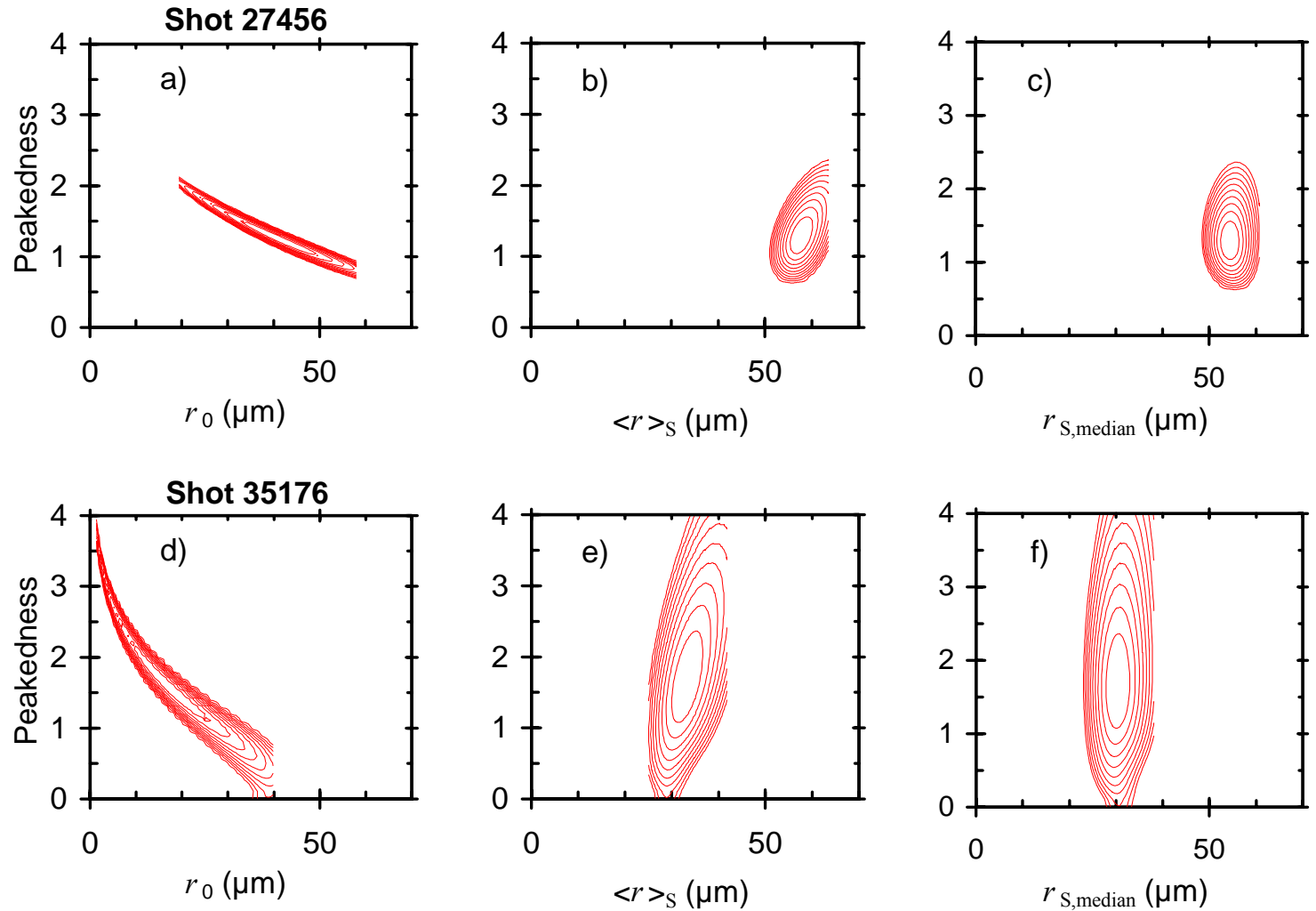

FIG. 8. (Color online) Contours of total $\chi^{2}$ for fits to two real data sets as a function of the shape parameter $p$ and either the $1 / \mathrm{e}$ radius $r_{0},\langle r\rangle_{\mathrm{S}}$, or $r_{S \text {, median. The upper three plots }}$ correspond to shot 27456, while the lower plots correspond to shot 35176 . In each case, the contour levels correspond to $\chi_{\text {minimum }}^{2}+1, \chi_{\text {minimum }}^{2}+2, \ldots$. 

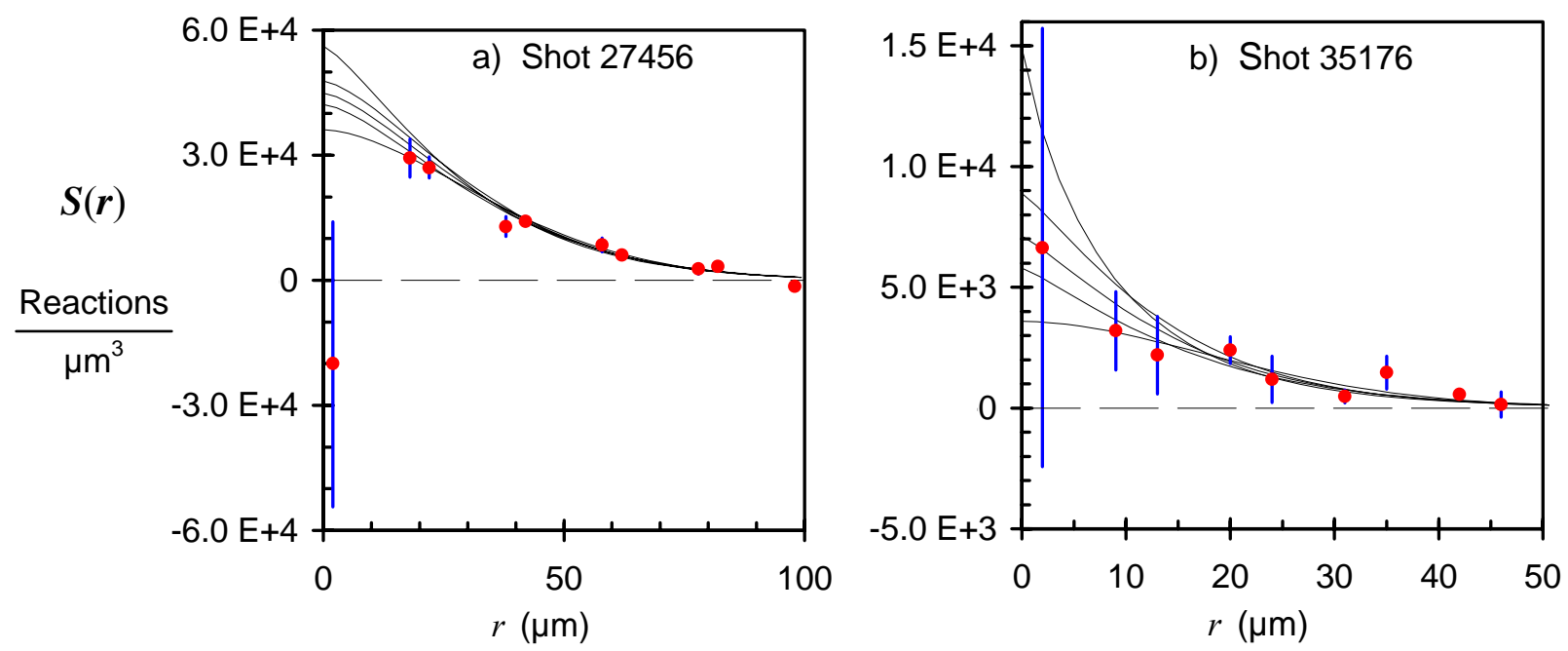

FIG. 9. (Color online) Results of applying the direct inversion method to data from shots 27456 (a) and 35176 (b). The plotted data points with error bars result from application of Eq. 14 to the $\mathrm{d} N / \mathrm{d} R$ data shown in Figs. 1a and 1e, though the data were binned slightly differently [with radial bins at the detector equivalent to bins in the burn region of $20 \mu \mathrm{m}$ for (a) and $11 \mu \mathrm{m}$ for (b), the effective ratios of bin width to $R_{\text {burn }}$ were 0.37 and 0.41 , respectively]. The uneven spacing of the data points reflects the fact that values of $S(r)$ were calculated from $P(x)$ for both positive and negative $x$, and $S$ values at negative $r$ values were reflected to positive $r$. The fine lines correspond to the profiles shown in Figs. 1d and 1h, including the error envelope. 